\title{
Numerical Taxonomy of Old World Phlebotominae (Diptera: Psychodidae). 1. Considerations of Morphological Characters in the Genus Phlebotomus Rondani \& Berté 1840
}

\author{
Philippe Rispail/ ${ }^{+}$, Nicole Léger* \\ Laboratoire d'Ecologie Médicale et Pathologie Parasitaire (Pr J-P Dedet), Faculté de Médecine, \\ 163 rue Auguste-Broussonet, 34090 Montpellier, France *Laboratoire de Parasitologie, Faculté de Pharmacie, \\ 51 rue Cognacq-Jay, 51090 Reims CEDEX, France
}

\begin{abstract}
Numerical analyses (correspondence analysis, ascending hierarchical classification, cladistic approach) were applied to the morphological characters of the adults of the genus Phlebotomus Rondani $\&$ Berté 1840. They confirm the reliability of the classic classifications, and also redefine the taxonomic and phylogenetic position of certain taxa. Thus, Spelaeophlebotomus Theodor 1948, Idiophlebotomus Quate \& Fairchild 1961 and Australophlebotomus Theodor 1948 deserve generic rank. Among the vectors of leishmaniasis, the subgenus Phlebotomus Rondani \& Berte 1840 is probably ancient. The results attribute an intermediate taxonomic and phylogenetic position to the taxa Euphlebotomus Theodor 1948 and Anaphlebotomus Theodor 1948, and reveal the probable artificial nature of the latter. The comparatively large numbers of species of subgenera Paraphlebotomus Theodor 1948, Synphlebotomus Theodor 1948 and, above all, Larroussius Nitzulescu 1931 and Adlerius Nitzulescu 1931, suggest that they are relatively recent. The development of adult morphological characters, the validity of their use in taxonomy and proposals for further studies are discussed.
\end{abstract}

Key words: Old World sand flies - Phlebotomus - Psychodidae Phlebotominae - numerical taxonomy

Taxonomy of the genus Flebotomus, created by Rondani and Berté (Rondani 1840) and emended to Phlebotomus by Loew (1845), has relied on a small number of adult characters considered separately but on a priori grounds given discriminant values (monothetic taxonomy). During more a century, the genus has been subdivided into an increasing number of subgenus (Table I). In 1982, Lewis subdivided Phlebotomus into 11 subgenera to which Abonnencius Ubeda-Ontiveros et al. 1982 and Transphlebotomus Artemiev \& Neronov 1984 were later added. The former was synonymised with Anaphlebotomus Theodor 1948 by Lane and Alexander (1988). Some authors gave generic rank to Spelaeophlebotomus Theodor 1948, Idiophlebotomus Quate \& Fairchild 1961 and Australophlebotomus Theodor 1948. There is some uncertainty as to the appropriate rank for these taxa and their phylogeny, and the evolutionary direction of the characters used by systematists.

\footnotetext{
${ }^{+}$Corresponding author. Fax: +33-4-67-63.0049. E-mail: parasito@sc.univ-montp1.fr

Received 26 March 1998

Accepted 29 July 1998
}

Numerical polythetic taxonomy simultaneously takes into account numerous characters without according any discriminant value, using advancements in information technology. Phenetic analysis expresses the inter-group relationships by means of factorial graphs and dendrograms. Cladistic analysis ends in the construction of cladograms, permitting the erection of filiation hypotheses and judgement of the direction of development of the characters.

\section{MATERIALS AND METHODS}

Selection of taxa - For the numerical analysis, specific taxa were chosen as the operational taxonomic units (OTU) (Sokal \& Sneath 1963). An examination of the available specimens permitted us to select discriminant characters. Lewis (1982) considered that the genus Phlebotomus Rondani $\&$ Berté 1840 was composed of about 110 specific or infra-specific taxa, unevenly grouped into 12 subgenera. Four arguments led to the elimination of certain taxa from the numerical analysis: validity does not seem to be totally established on solid bases (morphological as well as systematic); subspecific position is probable; inevitable constraints on the coding of the characters in closely related taxa; finally, and above all, a crippling imbalance in the calculation tables caused by missing data 


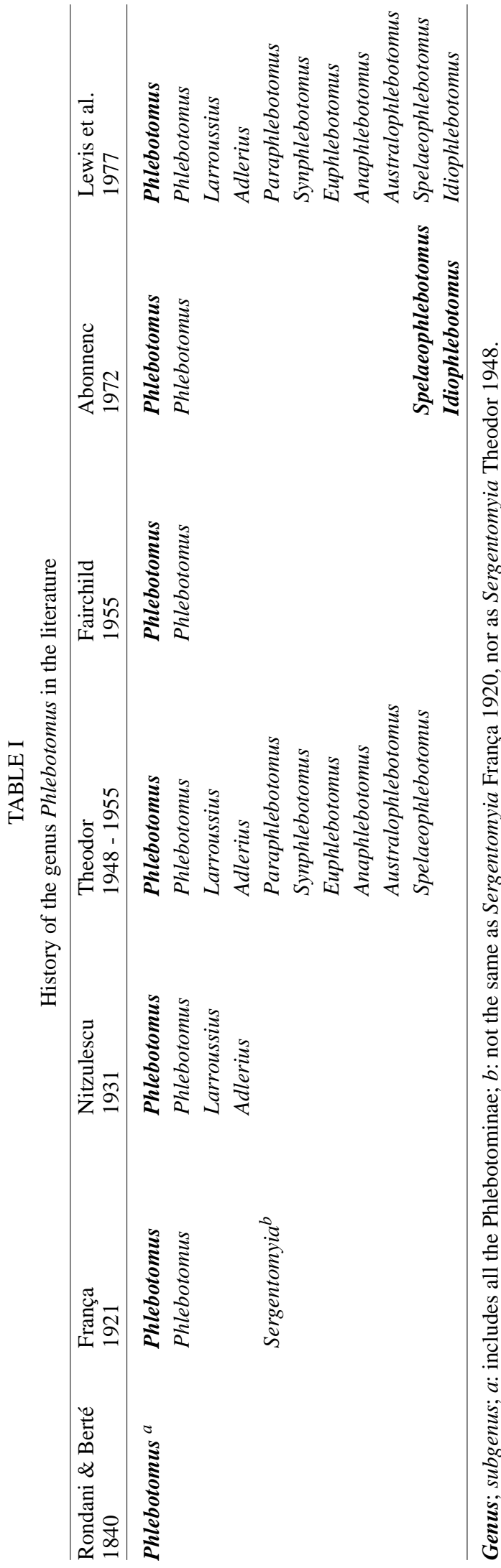

(incomplete description, species of which only one sex is known). Because of the latter the following taxa were eliminated: P. (Idiophlebotomus) sejunctus Quate 1965, P. (Id.) teshi Lewis 1978 and P. (Id.) tubifer Lewis \& Lane 1976; P. (Australophlebotomus) acuminatus Lewis \& Dyce 1982, P. (Au.) buccinator Fairchild 1952, P. (Au.) papuensis Fairchild 1952, P. (Au.) pexopharynx Fairchild 1952 and $P$. (Au.) trifilis Quate \& Quate 1967; P. (Synphlebotomus) katangensis Bequaert $\&$ Walravens 1930 and P. (Sy.) taylori Davidson 1982; P. (Larroussius) betisi Lewis \& Wharton 1963, P. (La.) chadlii Rioux, Juminer \& Gibily 1966, P. (La.) fantalensis Lewis, Minter \& Ashford 1974 and $P$. (La.) mariae Rioux, Croset, Léger \& Bailly-Choumara 1974; P. (Euphlebotomus) autumnalis Artemiev 1980 and $P$. (Eu.) caudatus Artemiev 1979; P. (Anaphlebotomus) somaliensis Abonnenc, Adam \& Bailly-Choumara 1959. Since our work begun, the female of P.(L.) fantalensis Lewis, Minter \& Ashford 1974 has been described. Three new species of the subgenus Larroussius have been named: $P$.(L.) lengi Zhang, He \& Ward 1994, P.(L.) ashfordi Gebre-Michael \& Lane 1996 and P.(L.) mireillae Killick-Kendrick et al. 1997. More, P.(L.) elgonensis Ngoka, Madel \& Mutinga 1975 has been taken reinstated (Killick-Kendrick et al. 1993).

Eventually 85 OTU's were retained. A preliminary cladistic analysis carried out jointly with phenetic analysis resulted in the establishment of the evolutionary direction and assessment of the validity of the characters. Twenty-three characters had to be eliminated, making the specific coding of each of the 85 taxa impossible, therefore 26 OTU's had to be excluded.

Choice of characters and definition of states The arguments of choice were: availability for all the taxa, absence of redundancy, clear definition of the states of characters and the stability of these states at a taxonomic level. Quantitative characters were, for the most part, excluded because of their variability. The description of the 85 taxa required the use of 63 characters and 176 character states (Table II).

Numerical analysis - Following the tabulation of character states for each OTU (Table III), analyses were carried out using an Olivetti M380 XP1 computer.

Phenetic analysis - Programmes were developed from those of the algorithm Analyse de Données of the software Biomeco 2.0 package (Groupe Biométrie CEPE/CNRS, Montpellier). Similarity tables of distance indices (Jaccard 1908) were transformed into multidimensional scatter diagrams, reduced to two dimensions by factor analysis, or into dendrograms, by ascending hierarchi- 
TABLE II

Numerical taxonomy of genus Phlebotomus Rondani \& Berté 1840 - 63 characters and their 176 states

Aa: antennal segments with two ascoids (female)

$\mathrm{Ab}$ : presence of two ascoids on antennal segment III (male) .. yes (P) [0] / no [1]

Ac: last antennal segment with two ascoids (male) XV (P) [0] / XIV [1] / XII or XIII [2] / IX, X or XI [3] / VII or VIII [4] / V [5]

Ad: antennal papillary formula (both sexes) 1/III-IV-V [1] / 1/III-IV [2] / 1/IV-V [3]/ other [4]

Pa: longest palpal segment $5[0] / 3[1]$

$\mathrm{Pb}$ : presence of spatulate sensillae on the palps no $(\mathrm{P})[0] /$ yes $[1]$

$\mathrm{Xa}$ : presence of cibarial armature (female) no (or rudimentary) [0]/ strong teeth (but not arranged in a palisade) [1]

$\mathrm{Xb}$ : female pharyngeal armature . rudimentary (a few ridges) [1] / intermediate (spines or small teeth) [2] / well developed (large teeth, scales) [3]

$X c$ : anterior expension of the pharyngeal armature (if intermediate) slight [1] / considerable [2]

Ta: anterior-inferior mesanepisternal hairs or scales

Wa: level of fork of fourth wing vein .... present $(\mathrm{P})[0] /$ absent [1]

Wb: ratio wing width/gamma

$\mathrm{Ba}$ : presence of a basal lobe on the coxite (\#) far from rm [0] / at level of rm [1]

$B b$ : morphology of basal lobe less than 3.5 [1] / equal or greater than 4 [2] no $(\mathrm{P})[0] /$ yes $[1]$ large but little prominent [1] / short with few short bristles [2] / long with long bristles [3]

$B c$ : morphology of the head of a long basal lobe rounded and symmetrical [1] / narrow and asymmetrical, curved down [2] / long and asymmetrical [3]

$B d$ : location of sockets bristles on a long basal lobe extremity only [1] / both at the tip and the distal area of the ventral side [2]

Be: number of bristles on basal lobe fewer or equal to 20 [1] / 25 - 35 [2] / around 80 [3]

Bf: presence of a tuft of bristles on the coxite no $(\mathrm{P})[0] /$ yes [1]

$\mathrm{Bg}$ : position of tuft of bristles on coxite subapical [1]/ median [2]

$B h$ : average number of bristles on the coxal tuft 20 or fewer [1] / 20 - 40 [2] / 40 - 60 [3] / 60 - 100 [4]

Ga: morphology of style / 100 - 150 [5] / more than 150 [6] ovoid and short [1] / medium length, narrow [2] / cylindrical, narrow, very long (> $300 \mu \mathrm{m})$ [3]

$G b$ : ratio length/width of style around 3 [1] / around 4 [2] / 5 - 7 [3] / 8 - 10 [4] / greater than 12 [5]

$G c$ : ratio length of coxite/length of style.... less than $1.5[1] / 1.5-2$ [2] / greater than 2 [3]

$\mathrm{Gd}$ : presence of one or several non-deciduous bristles on style . no $(\mathrm{P})[0] /$ yes $[1]$

Ge: number of spines on style three [3] / four [4] / five [5]

Gf: spines on style short [1] / long [2]

$\mathrm{Gg}$ : if five, distribution of spines on style three terminal, two median [1] / two terminal, three median [2]

$G h$ : if three terminal and two median, distance between basal and central spines

less than distance between central and distal spines [1] / equal [2] / greater [3]

Gi: position of terminal spines on style if two terminal and two median

both apical [1] / one apical, one subapical [2]

$\mathrm{Gj}$ : if three, distribution of spines on style two terminal, one median [1] / one terminal, two median [2]

Ca: morphology of parameres simple [1] / bilobed, with dorsal or ventral process [2] / trilobed (two process or one process and one tubercle) [3]

$\mathrm{Cb}$ : if paramere trilobed, relative lengths of lobes equal or subequal [1] / distinctly inequal [2]

Cc: shape of apex of parameres rounded or pointed [1] / truncate, squared or hocked

$\mathrm{Cd}$ : presence of spines on parameres [2] / flat and eliptical (spatulate) [3] no $(\mathrm{P})[0] /$ yes $[1]$

La: presence of spines on lateral lobes no (P) [0] / yes (spatulate or specifically shaped apical spines) [1].

Lb: if lateral lobe with spines, number of spines two (exceptionally three) [1] / more than three [2]

$L c$ : ratio length of lateral lobe / length of coxite distinctly less than 1 [1]/ around 1 [2]

/ distinctly greater than 1 [3] 
Va: shape of aedeagus short and conical [1] / long and digitiform [2] / very short, rudimentary [3] / specific shape [4]

$\mathrm{Vb}$ : if aedeagus short and conical, shape of apex blunt or truncate [1] / recurved [2] / pointed [3]

$\mathrm{Vc}$ : if aedeagus long and digitiform, diameter of valves uniform, parallel sided [1] / decreasing from base to tip [2]

$V d$ : if aedeagus long and digitiform, shape of apex rounded, blunt [1] / claviform [2] / with terminal

$V e$ : if aedeagus long and digitiform, and apex pointed, symmetry of apex bulb [3] / pointed [4] / transparent [5] / bifid [6] axial [1] / bilateral (bevelled) [2]

$V f$ : if aedeagus long and digitiform, and apex pointed and bevelled, orientation of bevel lateral-internal [1] / infero-internal [2] / ventral [3] / dorsal [4] / directed $90^{\circ}$ upwards, like a foot [5]

$V g$ : if aedeagus long and digitiform, and apex bifid, shape of tip

two spiniform branches [1] / one branche spiniform, the other rounded [2]

Vh: presence of spines on penis tips no $(\mathrm{P})[0] /$ yes $[1]$

$V i$ : type of spines on penis tips one - three lateral subapical spines [1] /medio-ventral denticles [2] / dorso-apical denticles [3] / a long lateral spine (between aedeagus and paramere) [4]

$\mathrm{Vj}$ : if aedeagus long and digitiform, with subterminal less than $10 \mu \mathrm{m}$ [1] / $10-20 \mu \mathrm{m}$ [2] / around tubercle, distance of tubercle from apex: $25 \mu \mathrm{m} \mathrm{[3]} \mathrm{/}$ 30 - $35 \mu \mathrm{m}[4] /$ greater than $40 \mu \mathrm{m}[5]$

$V k$ : ratio aedeagus length/coxite length

Fa: presence of intra-abdominal rods less than 0.4 [1] / $0.4-0.6$ [2] / greater than 0.6 [3]

$\mathrm{Fb}$ : shape of genital pump no [0] / yes [1]

small or hypotrophic [1] / normal [2] large or hypertrophic [3]

Fc: ratio length of genital filaments/length of pump less than 3 [1] / 3 - 5 [2] / 6.5 - 9 [3] / equal or greater than 9.5 [4]

$F d$ : modification of tip of genital filaments no $(\mathrm{P})[0] /$ yes $[1]$

Sa: structure of wall of spermathecal reservoir

$S b$ : if spermathecal reservoir ornamented, type of ornamentation clearly segmented, annulate [1] / with folds striations, incomplete segmentation [2]

$S c$ : if spermathecal reservoir segmented, mean number of segments less than $13[1] / 13-20$ [2] / $21-29$ [3] / 30 or more [4]

$S d$ : if spermathecal reservoir segmented and mean number of segments less than 13, number of segments ... 2 [1] / 3 - 6 [2] / 7 - 12 [3]

Se: morphology of spermathecal reservoir .... cylindrical, tubular [1] / conical [2] / fusiform [3] / subspherical [4] / saccate, globular or of distinctive shape [5]

Sf: terminal segment of spermathecae undifferentiated $(\mathrm{P})[0] /$ differentiated, swollen, campanulate [1]

$\mathrm{Sg}$ : if spermathecae segmented, neck short or absent (P) [0] / long and digitiform [1]

$S h$ : if spermathecal reservoir smooth, shape of reservoir. without well defined capsule or demarcation between reservoir and duct [1] / with well defined capsule [2]

Si: diametre of spermathecal ducts mostly uniform, any dilatation only at base (P) [0]

/ dilated over much of their length [1]

Sj: opening of spermathecal ducts separate [1] / by a common duct [2]

Sk: length of spermathecal ducts average (P) [0] / exceptionally long [1]

italics: characters secondarily rejected; $(\mathrm{P})$ : presumed plesiomorphic state; (\#): terminology of male genitalia follows Abonnenc (1972).

cal classification. In the latter, cluster analysis used intermediate linkage, and, in the absence of additional information, linkages at less than 50\% similarity were considered as random.

Cladistic analysis - The MIX algorithm (Wagner parsimony), extracted from the PHYLIP programme, distributed by Felsenstein (1978,
1985), was used. This allows differentiation between occasional missing values and indeterminations, expressing characters of which no state applies to the coded taxon. Bias introduced by concentration of characters relating to a single organ is compensated by ascribing reduced weight to dependent characters. In the absence of sufficiently 
TABLE III

Numerical taxonomy of the genus Phlebotomus. Characters state matrix. Codes as in Table I

\begin{tabular}{|c|c|c|c|c|c|c|c|c|c|c|c|c|}
\hline & $\begin{array}{l}\text { A A } A \text { A } \\
\text { a b } c \text { d }\end{array}$ & $\begin{array}{l}\mathrm{P} P \\
\mathrm{a} \mathrm{b}\end{array}$ & $\begin{array}{l}X X X \\
\mathrm{a} \quad \mathrm{b} c \\
\end{array}$ & $\begin{array}{l}\mathrm{T} \\
\mathrm{a}\end{array}$ & $\begin{array}{l}\text { WW } \\
\text { a b }\end{array}$ & $\begin{array}{l}\mathrm{B} B B B \mathrm{~B} \mathrm{~B} \mathrm{~B} B \\
\mathrm{a} b c d \mathrm{e} \mathrm{f} h\end{array}$ & $\begin{array}{l}\text { GGGGGGGG G G } \\
\text { a } b c d \text { ef } g h \mathrm{i} \mathrm{j}\end{array}$ & $\begin{array}{l}\mathrm{CC} \mathrm{CC} \\
\mathrm{a} b \mathrm{~cd}\end{array}$ & $\begin{array}{l}\mathrm{L} L L \\
\mathrm{a} \mathrm{b} c\end{array}$ & $\begin{array}{l}\mathrm{VVV} V V V V \mathrm{~V} V \mathrm{~V} V \\
\mathrm{abc} d e f g \mathrm{~h} i \mathrm{j} k\end{array}$ & $\begin{array}{l}\text { F F F } F \\
\text { a b c } d\end{array}$ & $\begin{array}{l}\mathrm{S} S S S \operatorname{SS} S \operatorname{S} \\
\mathrm{a} b c d \mathrm{e} \mathrm{f} h i \mathrm{j} \\
\end{array}$ \\
\hline P.(Ph.)bergeroti & & 01 & & 0 & 1 & & & & & & & \\
\hline P.(Ph.)duboscqi & 1001 & 01 & $3 X$ & 0 & 1 & $12 \times \times 1$ & & & & & & \\
\hline P.(Ph.)papatasi & 1001 & 1 & $3 X$ & 0 & 1 & $2 x$ & X X & & & X $0 \times X 1$ & & $111310 \times X 010$ \\
\hline P.(Ph.)salehi & & 01 & $3 x$ & 0 & 01 & & $5112 \times X$ & & & $11 \times \times X X X 0 \times X 1$ & & $20 \times \times 010$ \\
\hline P.(Pa.)alexandri & 1001 & 01 & $3 X$ & 0 & 1 & & $X 2 X$ & & & $X 1$ & & \\
\hline P.(Pa.)andrejevi & 1001 & 01 & $3 X$ & 0 & 01 & $131120 X X$ & $123042 \times X 2 X$ & & & 12 X X X X X 0 X X 1 & & 111 \\
\hline P.(Pa.)caucasicus & 1 & 01 & $3 X$ & 0 & 01 & & & & & K X 1 & & 10 \\
\hline P.(Pa.)chabaudi & & 01 & $3 x$ & 0 & 01 & & & & & K 1 & & \\
\hline P.(Pa.)jacusieli & & 01 & $03 \mathrm{X}$ & 0 & 01 & & & & & $X 1$ & & \\
\hline P.(Pa.)kazeruni & 1 & 01 & $3 X$ & 0 & 01 & 101 & $X X 2 X$ & & & X X 1 & & 10 \\
\hline P.(Pa.)mal & & 01 & $3 X$ & 0 & 01 & & $1 \mathrm{X}$ & & & $X 1$ & 0 & 10 \\
\hline P.(Pa.)mongolensis & & 01 & $03 X$ & 0 & 01 & & $2 X$ & & & X 1 & & \\
\hline P. $(P a)$. & & 01 & $03 \mathrm{X}$ & 0 & 01 & & $2 X$ & & & X 1 & & 10 \\
\hline$P .(P a$ & 1 & 01 & $3 X$ & 0 & 01 & & $\mathrm{X}$ & & & X 1 & & \\
\hline & & & & 0 & & & & & & & & \\
\hline$P .(P a$. & & 01 & $03 \mathrm{X}$ & 0 & 01 & & & & & & & \\
\hline P. $(S)$ & 1 & 0 & 21 & 0 & 01 & & $x X$ & & & X 1 & & \\
\hline$P .(S y$. & & 01 & 021 & 0 & 01 & & X X & & & $X 1$ & & 10 \\
\hline P. (S & & & $03 X$ & 0 & 01 & & $X X$ & & & & & \\
\hline$P .(S)$ & & 01 & $01 \mathrm{X}$ & 0 & 01 & & X X & & & X 1 & & 10 \\
\hline$P .(S)$ & & 01 & 021 & 0 & 01 & & $X X$ & & & $X 1$ & & 10 \\
\hline$P .(S y)$. & & 01 & 021 & 0 & 01 & & $X$ & & & & & \\
\hline$P .(S y)$. & & 01 & 021 & 0 & 01 & & & & & $X 1$ & & 10 \\
\hline P.(L.) & 1041 & 01 & 021 & 0 & 01 & 22 & X X & & & X 1 & & 10 \\
\hline$P .(L) c$. & & 01 & & 0 & 0 & & & & & & & \\
\hline P.(L.)gibiensis & & 01 & 021 & 0 & 01 & 0 X X X X 121 & $522 \times X X$ & $2 \times 10$ & & $2 \times 11 \times X X 0 X X 2$ & 0220 & $1113101 \times 110$ \\
\hline P.(L.)guggisbergi & 1001 & 01 & 021 & 0 & 01 & $11 \mathrm{XX} 10 \mathrm{XX}$ & $2230522 \times X X$ & $2 \times 10$ & & $2 \times 141 \times X 0 \times X 1$ & 0220 & $112 X$ \\
\hline P.(L.)kandelakii & & 01 & 021 & 0 & 01 & & & & & & & \\
\hline P.(L.)keshishiani & & 01 & 021 & 0 & 01 & 121 & & & & X 2 & 20 & 112 \\
\hline P.(L.)langeroni & 1021 & 01 & 021 & 0 & 01 & $X 121$ & 522 X X X & $2 \times 10$ & & $2 \times 2421 \times 0 \times X 2$ & 0220 & $1113101 \times 010$ \\
\hline P.(L.)longicuspis & 1041 & 01 & 021 & 0 & 01 & $X 121$ & $522 \times X X$ & $2 \times 10$ & 2 & $2 \times 24$ & 0220 & $\mathrm{X} 010$ \\
\hline P.(L.)longipes & 1041 & 01 & & 0 & 01 & & $X X$ & & & & 0220 & $112 X$ \\
\hline P.(L.)major & 1041 & 01 & 021 & 0 & 01 & $X 122$ & $22 \times X X$ & $2 \times 10$ & & $\times 0 \times X 2$ & 0220 & $01 \times 020$ \\
\hline P.(L.)neglectus & 1041 & 01 & 022 & 0 & 01 & $0 \times X X X 122$ & $2330522 \times X X$ & $2 \times 10$ & $0 \times 2$ & $2 \times 13 \times \times \times 0 \times X 2$ & 0220 & $112 \times 101 \times 020$ \\
\hline
\end{tabular}


A A $A$ A P P XXX T WW BBBB B B B $\quad$ GGGGGGGGGG CC CC L L $L \quad$ VVV VVVVVVVV FFFF SSSSSSSSS

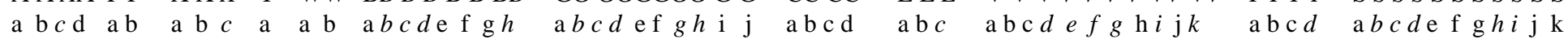

\begin{tabular}{|c|c|c|c|c|c|c|c|c|c|c|c|c|}
\hline P.(L.)notus & 041 & 01 & 022 & 0 & 01 & 0 X X X X 122 & $320522 \mathrm{XXX}$ & $2 \times 10$ & $0 \times 2$ & $2 \times 13 \times X X 0 \times X 2$ & 0220 & 11 \\
\hline P.(L.)orientalis & 041 & 01 & 21 & 0 & 01 & 0 X X X X 121 & 522 XXX & & & $2 \times 0 \times \times 2$ & & 10 \\
\hline P.(L.)pedifer & 41 & 01 & 21 & 0 & 01 & X X X X 122 & 22 XXX & & & $5 \times 0 \times \times 2$ & & \\
\hline P.(L.)perfiliewi & 001 & 01 & 21 & 0 & 01 & X X X X 121 & 320522 X X X & & & X 15 X X X 13 X 2 & 220 & X 010 \\
\hline (L.)perniciosus & 1041 & 01 & 21 & 0 & 01 & X X X X 121 & 2320522 XXX & X 10 & $X 2$ & $2 \times 26 \times X 10 \times X 2$ & 0220 & 10 \\
\hline (L.)smirnovi & & 01 & 21 & 0 & 1 & X X 122 & $22 \times X X$ & & K 2 & $\mathrm{~K} \times 2$ & & 20 \\
\hline & & 01 & 21 & 0 & 01 & XXXX X 123 & $522 \times$ X X & & & X X 0 X X 2 & 0220 & $x 020$ \\
\hline & & 01 & 1 & 0 & 1 & 22 & $22 x$ & & & $\mathrm{X} 2$ & 20 & \\
\hline (I transch & $s 1041$ & 01 & 21 & 0 & 01 & 0 X X X X 121 & 522 X X X & 0 & $\times 2$ & $2 \times 15$ & 220 & 10 \\
\hline (L.) & & 01 & $3 X$ & 0 & 01 & 22 & 22 X X X & & & $\times 2$ & 0 & 20 \\
\hline (L.)wui & & 01 & 21 & 0 & 01 & 122 & 23 & & & $\times 2$ & 220 & 20 \\
\hline$(T)$. & & 01 & $3 \mathrm{X}$ & 0 & 01 & 22 & $22 x$ & & 3 & X 2 & 220 & 20 \\
\hline (Ad.)angustus & & 01 & $X$ & 0 & 0 & 23 & 23 & & & 22 & 0 & 10 \\
\hline (Ad.)arabicus & & 01 & $3 X$ & 0 & 0 & 23 & 22 X X X & & 3 & 22 & 230 & 10 \\
\hline (Ad.)balcanicus & & 01 & $X$ & 0 & 0 & 25 & $22 \times X X$ & & & 22 & & \\
\hline vis & 1 & 01 & $\mathrm{X}$ & 0 & 0 & 21 & $22 x$ & & & 32 & 30 & 10 \\
\hline )chinensis & & 01 & $\mathrm{X}$ & 0 & & 22 & 23 & & & +2 & 30 & 10 \\
\hline atus & & 01 & $\mathrm{X}$ & 0 & 0 & 26 & $22 x$ & & & 22 & 0 & 10 \\
\hline epensis & & 01 & & 0 & & 23 & 2 & & & & & \\
\hline & & 01 & & 0 & 0 & 24 & $2 x$ & & & & & 10 \\
\hline & & 01 & & 0 & 0 & & & & & & & \\
\hline & & 01 & & 0 & 0 & 24 & 23 & & & & & 10 \\
\hline$i s$ & & 01 & $3 X$ & 0 & 0 & 24 & $22 \mathrm{X}$ & & & & & \\
\hline & & 01 & $3 X$ & 0 & 0 & 22 & $22 x$ & & & 52 & 30 & 10 \\
\hline & & & & 0 & 0 & 21 & $22 \times X X$ & & & & 0 & \\
\hline anicus & 1 & 01 & $3 \mathrm{X}$ & 0 & 0 & 22 & $22 \times X X$ & & & $2 X$ & 30 & 10 \\
\hline (E.)argentipes & & 01 & & 0 & 0 & X X & $22 \mathrm{XXX}$ & & & X X & 0210 & 20 \\
\hline P.(E.)kiangsuensis & & 01 & 21 & 0 & 0 & & & & & X 1 & 0211 & 20 \\
\hline P.(E.)mesghalii & 1 X X 1 & 01 & 021 & 0 & 01 & $X X$ & $22 \times X X$ & & & X 2 & 0220 & $12 x$ \\
\hline P.(E.)philippinensis & & 01 & & 0 & 01 & & $22 \mathrm{XXX}$ & & & X 2 & $02 \times 0$ & $12 \mathrm{Y}$ \\
\hline P.(E.)tumenensis & 1001 & 01 & 021 & 0 & 01 & X X & $22 \mathrm{XXX}$ & & & $11 X X$ & $02 \times 0$ & $10 \times 020$ \\
\hline (E.)yunshengen. & & 01 & 021 & 0 & 01 & & $22 \times X X$ & & & $X 1$ & & 111 \\
\hline P.(K.)newsteadi & 1032 & 01 & $03 \mathrm{X}$ & 0 & 01 & & $213 \times X$ & & & 11 X X X X X 14 X 2 & 0210 & $11 \times \times 020$ \\
\hline P.(An.)colabaensis & $1 \mathrm{X} X 1$ & 01 & 121 & 0 & 01 & $0 \times X \times X 0 \times X$ & $123042 \times X 2 X$ & $2 \times 10$ & $0 \mathrm{XX}$ & 13 X X X X X 0 X X X & $02 \mathrm{XX}$ & $112 \times 200 \times 021$ \\
\hline
\end{tabular}




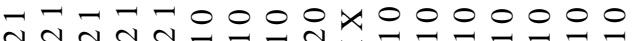

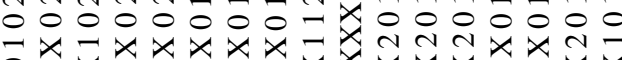
$00 x$ o $0 x x x x x x x x x x x$

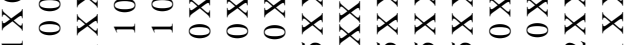

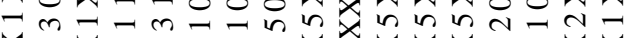
$\bar{x} \bar{x} \bar{x} \bar{x} \bar{x} \times \bar{x} \times \bar{x} \times \bar{x} \bar{x} \bar{x}$ $x a x+n x x x x x x x x x$

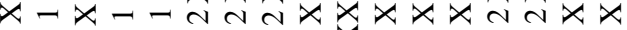
$0=0=I N I 0 x 0001000$

ox $x_{0} 000-1000000000$

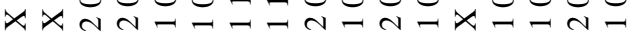

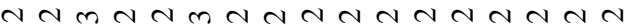
$0000000001-1-1-1-1$ $-N-F-\bar{x}-\bar{x}-2 n-c-c$ $\bar{x} x \bar{x} \bar{x} \times x \times x \times x \times x \times x$ $\forall \forall x x \forall x x x x x x x x x x x$ - - $00-00000000000000$ $x \times x \times x \times x \times x \times x \times x \times x x$ $x \times x \times x \times x \times x \times x \times x \times x \times x$ $x \times x \times x \times x \times x \times x \times x \times x \times x$ $x \times x \times x \times x \times x \times x \times x \times x \times x$ $x \times x \times x \times x \times x x=x x x x$ $=-=m x \times x=-d x x=c-1$ - - - - m m n- -

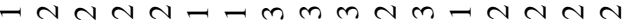
$x \times x \times x \times x \times x \times x \times x \times x \times x$ $0000000-000000000$

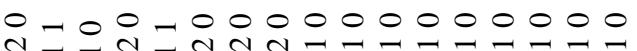
$x \sqrt{n} \times \vec{\sim} \times x \times x \times x \times x \times x \times x$ तm $m$ n

$x x x x x x-a x x-a x n x x+n$ $x-n d a x x x a d x x a x x d x$ $x \times x \times x \times x \times x \times x \times x \times x \times x$ $\sim x \times x \times x \times x \times x \times x \times x \sim x \times$

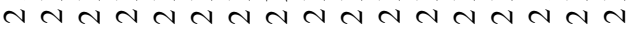
$n \forall \forall \forall \forall m m m \forall \forall m m \forall m n \forall m$ $00000000-1-000000$

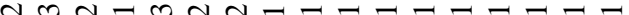
$m-m m-\pi-N n+t+n n+n n$

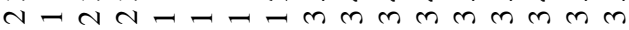
$x \times x \times x \times x x-x x-x-x$ $x \times x \times \sim x x x=x \times x=x=x$ $0000-0000-00-1-0=0$ $x \times x \times x \times x \times x \times x \times x \times x \times x$ $x \times x \times x \times x \times x \times x \times x \times x \times x$ $x \times x \times x \times x \times x \times x \times x \times x \times x \times x$ $x \times \times \times \times \times \times \times \times \times \times \times \times \times \times \times x$ 00000000000000000

- J্টি, $00000--1-\neg--\neg-1-1$

$x \times x-1 \times x \times x \times x \times x \times x \times x$

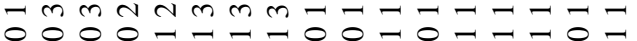
- - - - - - - -000000000 $000000001-1-1-1-1-1$ $=\overline{0}-\overline{0}-\bar{x}+x \times x \times x \times x=$ $-x m m n-1.0 m$ mo $0000000 x 00-1-00-00$

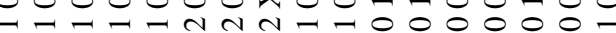

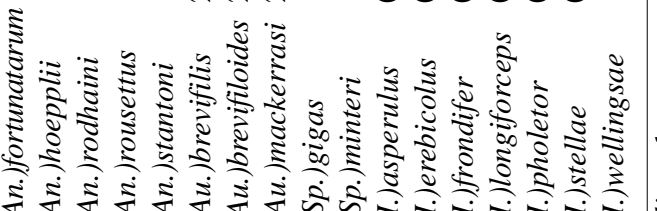

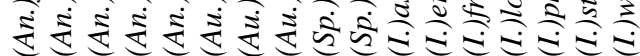

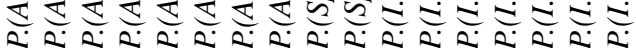

well known fossils to determine plesiomorphy, the supposed evolutionary direction of the greatest number of these characters, required to root the phylogenetic tree, was supported from studies of other Psychodidae (Hennig 1972). The construction of Wagner diagrams (Wagner 1961) used Manhattan distance (Legendre \& Legendre 1979), and took account simultaneously of the character states and the distances between OTU's.

\section{RESULTS}

\section{PHENETIC ANALYSIS}

The first axis of the correspondence analysis separates the species of the taxa Idiophlebotomus, Spelaeophlebotomus and Australophlebotomus from those of the others groups. The most important characters in this axis are the antennal formula in the female, the palpal formula and the morphology of the sensillae, the presence or absence of mesanepisternal setae, the shape of the wing, the structure and number of spines on the style, the presence or absence of intra-abdominal rods, and the morphology of the spermathecae. The second axis separates Paraphlebotomus and Synphlebotomus from Idiophlebotomus and Spelaeophlebotomus. The important characters here are the male genital structure (basal lobe, style, paramere, aedeagus) and the presence or absence of segmentation on the spermathecae. As with the correspondence analysis, ascending hierarchical classification (Fig. 1) confirms generally accepted subgenera. While the subgeneric level overall is approximately $60 \%$ of similarity, the species of the subgenera Phlebotomus, Paraphlebotomus, Synphlebotomus, Adlerius and Larroussius join at a high level of similarity (70-80\%). The species of the other taxa are further one from the other. Paraphlebotomus and Synphlebotomus come together early, then are joined by Phlebotomus. P. (Transphlebotomus) mascittii attaches to Adlerius before the major union with Larroussius. The numerous distinctive characters of $P$. (Euphlebotomus) yunshengensis integrate $P$. (Kasaulius) newsteadi into the block of Euphlebotomus. The three African species of Anaphlebotomus join to Euphlebotomus, while the three Asiatic species are completely separate. The taxa Spelaeophlebotomus, Idiophlebotomus and Australophlebotomus separate very late, at a level of similarity without any significance.

\section{CLADISTIC ANALYSIS - PHYLOGENETIC HYPOTH- ESES AND DEVELOPMENT OF THE CHARACTERS}

First phylogenetic hypothesis - On the most parsimonious Wagner's tree achieved (Fig. 2), the species of the taxa Spelaeophlebotomus and Idiophlebotomus are the first to form a group. The 


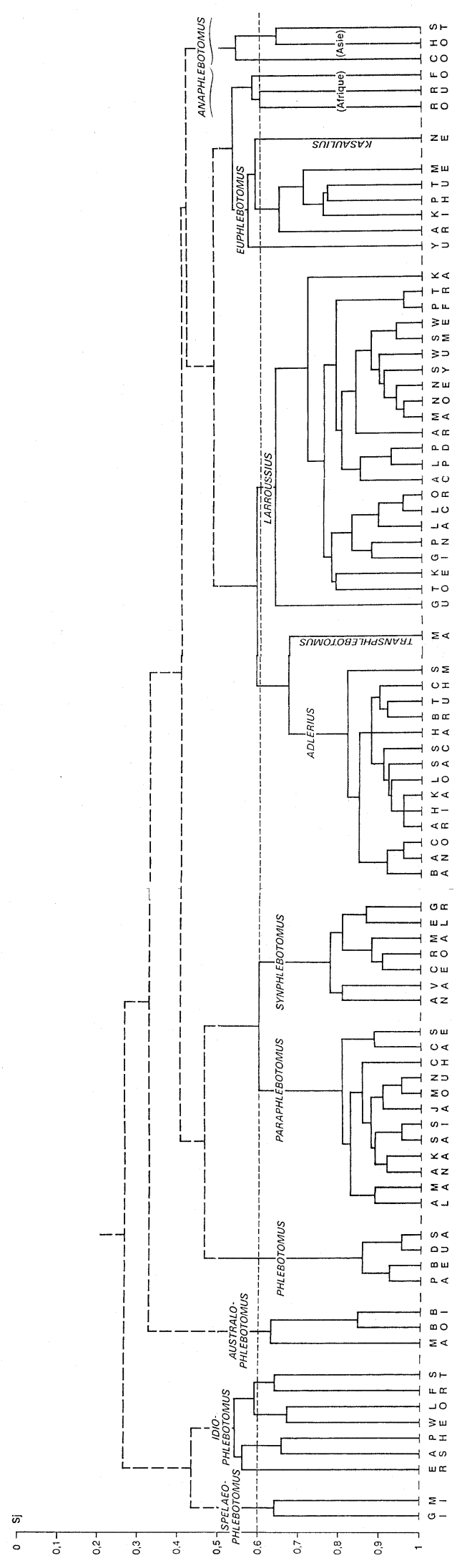

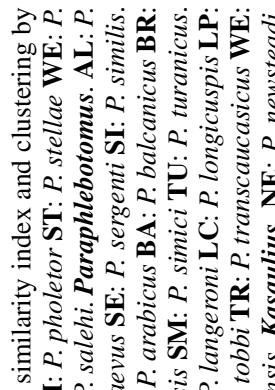

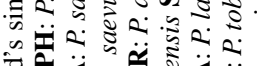

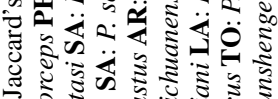

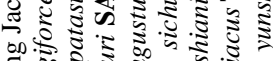

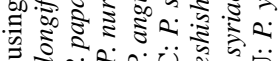

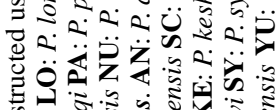

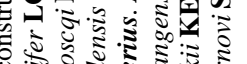

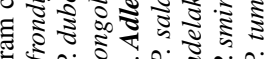

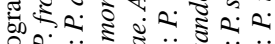

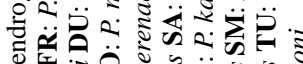

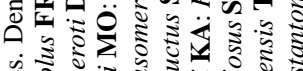

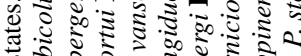

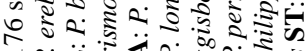

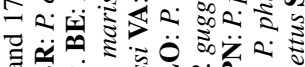

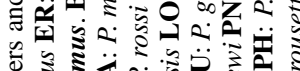

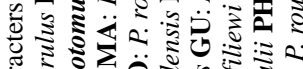

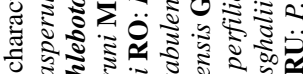

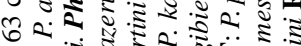

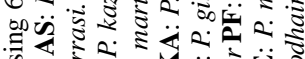

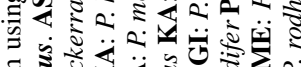

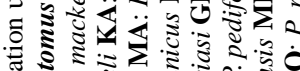

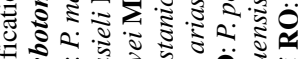

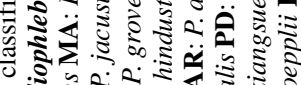

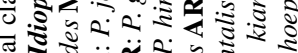

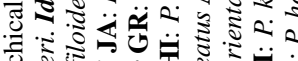

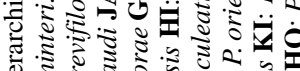

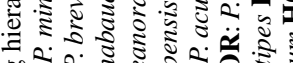

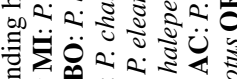
ठै.

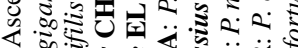

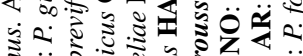

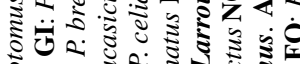

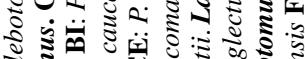

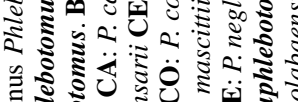

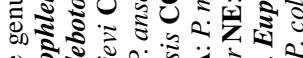
सं

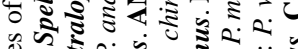

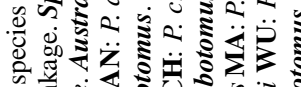
की

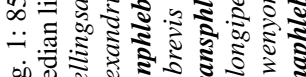

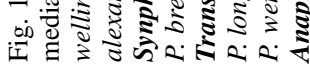




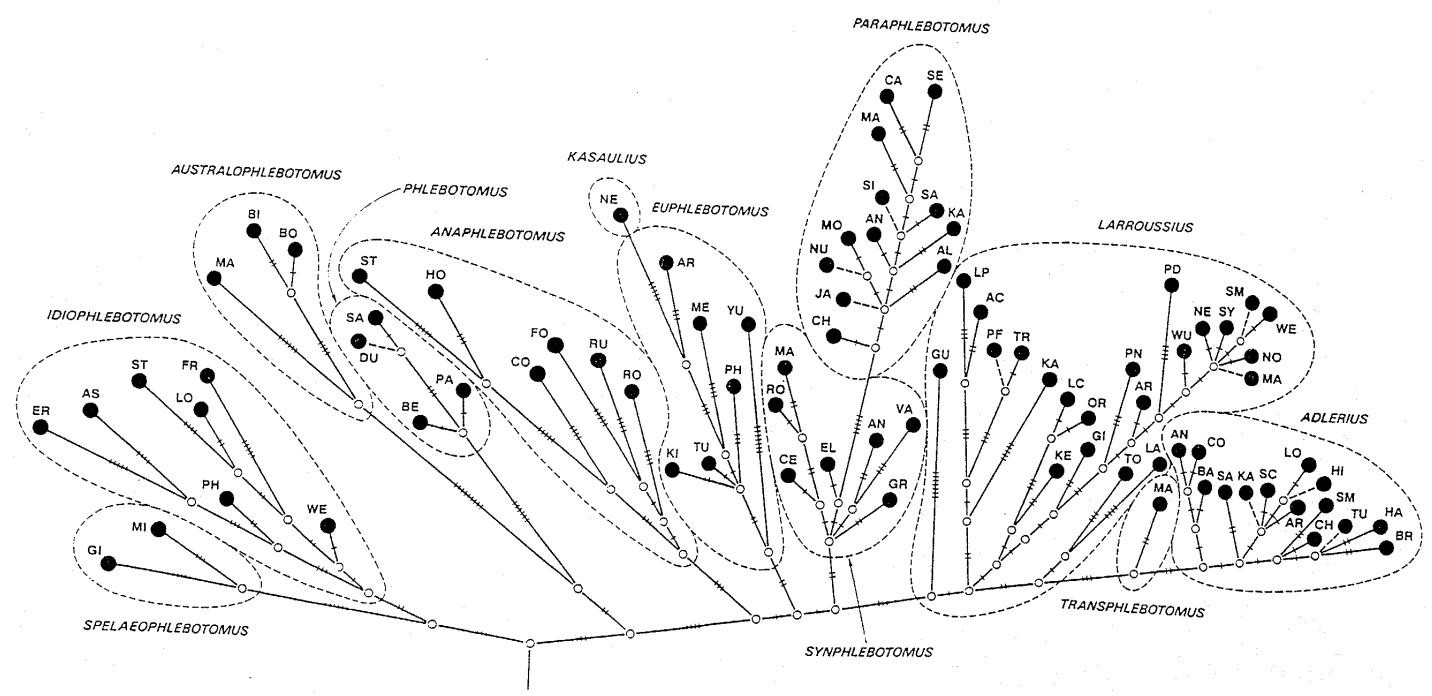

Fig. 2: 85 species of the genus Phlebotomus. Cladistic analysis using 63 characters and 176 states. Most parsimonious cladogram (317 steps). Symbols are the same as in Fig. 1. Bars indicate the number of steps.

species of the subgenera Australophlebotomus and Phlebotomus quickly diverge from an early common branch. The next branch contains the species of the subgenus Anaphlebotomus. The three African species diverge very quickly from the Asiatic branch. The branch Kasaulius comes from the Euphlebotomus branch. The first node in the branch appearing afterwards would be a hypothetical common ancestor of Synphlebotomus and Paraphlebotomus. The terminal branches illustrate the abundant specific diversification within the subgenera Larroussius, Transphlebotomus and Adlerius, which are probably the most developed.

Evolution of the characters - One of the major interests of cladistic analysis is the consideration of each character individually: definition of its states, probable direction of development and the validity of its use. To investigate this, each one of the 63 characters used was followed from the root of the tree to its last branches. The principal conclusions from this study are: Antennae - Accepting the presence of two ascoids on antennal segments III to XVI in the female, as plesiomorphic by analogy with the other Psychodidae, Idiophlebotomus appears to be an ancestral group, Australophlebotomus seems be very well developed and Spelaeophlebotomus is intermediate. On the other hand, in the male, only three species of the genus Idiophlebotomus show the derivative state "absence of two ascoids on the third segment", a condition which disagrees with that of the antennal formula in the female, diminishing the cladistic interest of this character. The papillary formula of the antennae 1/III-IV-V occurs only in the four species of the branch Phlebotomus and in $P$. (Australophlebotomus) brevifiloides. However, the lack of data for the majority of the species of the taxa Spelaeophlebotomus and Idiophlebotomus prevents us from confirming that this state is really ancestral. Palps - The plesiomorphy of the absence of spatulate sensillae found only in Spelaeophlebotomus and Idiophlebotomus has not been formally confirmed, but is compatible with the structure of the phylogenetic tree. Palpal segments 3 longer than the others are seen in the group Spelaeophlebotomus - Idiophlebotomus which is probably ancestral. Thus this state seems to be plesiomorphic. Cibarium - The cibarium of the female is armed with teeth only in Australophlebotomus and the majority of Idiophlebotomus. The presence of a well developed armature in the neighbouring genera Sergentomyia and Lutzomyia leads us to believe that the analysis of this character should be done at a family level. Pharynx - The evolution of the pharyngeal armature seems to be in the direction of the development of structures from simple ridges towards large teeth with forward expansion. As the most well developed state is always found at the end of the branch, the development of the pharyngeal armature is probably an adaptative character as, perhaps, is the cibarial armature. Thorax - Following the examples of the American taxa Hertigia and Warileya, alone within the genus Phlebotomus, the species of the taxa Spelaeophlebotomus and Idiophlebotomus do not have an antero-inferior cluster of mesanepisternal bristles or scales. If one considers these groups as more primitive than the others, the hypothesis of a 
development towards the disappearance of this cluster must be submitted to further investigation. In fact the discussion essentially concerns whether or not these two taxa belong in the genus Phlebotomus. Wings - In Spelaeophlebotomus the fourth vein $(\mathrm{M} 1+\mathrm{M} 2)$ branches at the level of the radio-medial (rm). In all the other taxa, this branch is more or less distant from rm. Relatively short and wide wings are characteristic of the primitive groups, and the hypothesis of evolutionary lengthening of "gamma" seems to be verified. A ratio "width of wing/gamma" equal to or greater than 4 is apparently an ancestral character present in the taxa Spelaeophlebotomus and Idiophlebotomus. Coxites - The coxites have a basal lobe in the Phlebotomus and the whole SynphlebotomusParaphlebotomus complex. Its origin might be separate in these two groups. It is plausible that this is a synapomorphic state. On the other hand, the absence of a cluster of bristles on the coxite seems to be a plesiomorphic state. These coxal bristles are sub-apical in the groups which seem to be the oldest (Spelaeophlebotomus, Idiophlebotomus and Phlebotomus). The presence of the coxal bristles, their medial position and increasing numbers of bristles are apparently developed states. Styles - A very long and narrow cylindrical style in Spelaeophlebotomus, Idiophlebotomus and Phlebotomus is probably a plesiomorphic character. The styles of Phlebotomites and Warileya are similar in shape. Furthermore, the morphology of the styles clearly contributes to the separation of Anaphlebotomus into two groups, one African, with wide styles of medium length, the other Asiatic with short ovoid styles. This short ovoid style, found in Australophlebotomus, the Asiatic Anaphlebotomus and Paraphlebotomus is probably an apomorphic state. This synapomorphy is possibly a case of parallel evolution of a functional character. The number of spines on the styles is a very important character for identification. Its evolutionary significance is more questionable, but the hypothesis of development towards a reduction in the number and terminalisation of these spines, by analogy with other Psychodidae, is plausible. Typical synapomorphy, only the four species of the subgenus Phlebotomus have small spines. Parameres - While all the species of each subgenus have the same type of paramere, the development of this character still remains undetermined. The phylogenetic reconstruction is not incompatible with the hypothesis of a simple ancestral paramere, and probably reflects the adaptation of this functional character. In the development of a trilobe paramere, one of the lobes appears originally to be clearly longer than the other two, and the respective lengths of the three lobes apparently have a tendency to level out. This hypothesis would reinforce that of a simple, generally long, ancestral paramere. Aedeagus - The short conical aedeagus present in the majority of the groups is perhaps a plesiomorphic character. The rudimentary aedeagus of Australophlebotomus, the long digitiform penis of Larroussius, Transphlebotomus, Adlerius, and of $P$. (Id.) erebicolus, and the distinctive valves of $P$. (Id.) frondifer could be considered as developed states. A short conical, blunt tipped aedeagus is probably the ancestral shape. Development seems to have been either towards a curved extremity (most of Paraphlebotomus, part of Idiophlebotomus) or, more rarely, towards a clearly pointed extremity. Spicules, denticules or spines on the tips are only found at the end of the branches in the subgenera Anaphlebotomus, Euphlebotomus, Kasaulius, and Larroussius. Intra-abdominal rods - Intra-abdominal rods are only present on the ancestral branch Spelaeophlebotomus - Idiophlebotomus; elsewhere, they also exist in the genus Warileya which is probably an ancient Neotropical group and favours the plesiomorphy of this state.

Genital pump and filaments - Exceptionally small or large genital pumps are apomorphic states. Development was presumably through the progressive increase in the ratio length of genital filaments / length of the pump. From the ancestral state "less than 3", it increases to "3-5" in Larroussius and Transphlebotomus, to "6.5-9" then "greater than 9.5" in Adlerius. Lateral lobes - The absence of spines on the lateral lobes is confirmed as a plesiomorphic state. The apomorphic state only concerns the apical spatulate spines of Phleboto$m u s$. The number of spines increases from two in $P$. bergeroti and $P$. papatasi to more than three in P. duboscqi and P. salehi. Spermathecae - Cylindrical spermathecae seem to be ancestral. The presence of ornamentation on the reservoir wall of the spermathecae is exclusive to the developed groups: only the taxa Spelaeophlebotomus, Idiophlebotomus and Anaphlebotomus contain the species with smooth spermathecae. In these groups, the evolution from smooth, poorly defined spermathecae towards spermathecae with a well defined capsule cannot be confirmed. In the species with ornamented spermathecae, clear segmentation has progressively diminished. The overall development would, therefore, be from the smooth spermathecae to the clearly segmented spermathecae and then to the pleated or ridged spermathecae. The separate opening of the spermathecae ducts is a plesiomorphic character. Common ducts occur at the end of the branches: in $P$. (Sp.) gigas, Anaphlebotomus, Kasaulius and Euphlebotomus (except $P$. yunshengensis), $P$. (T.) mascittii and the 


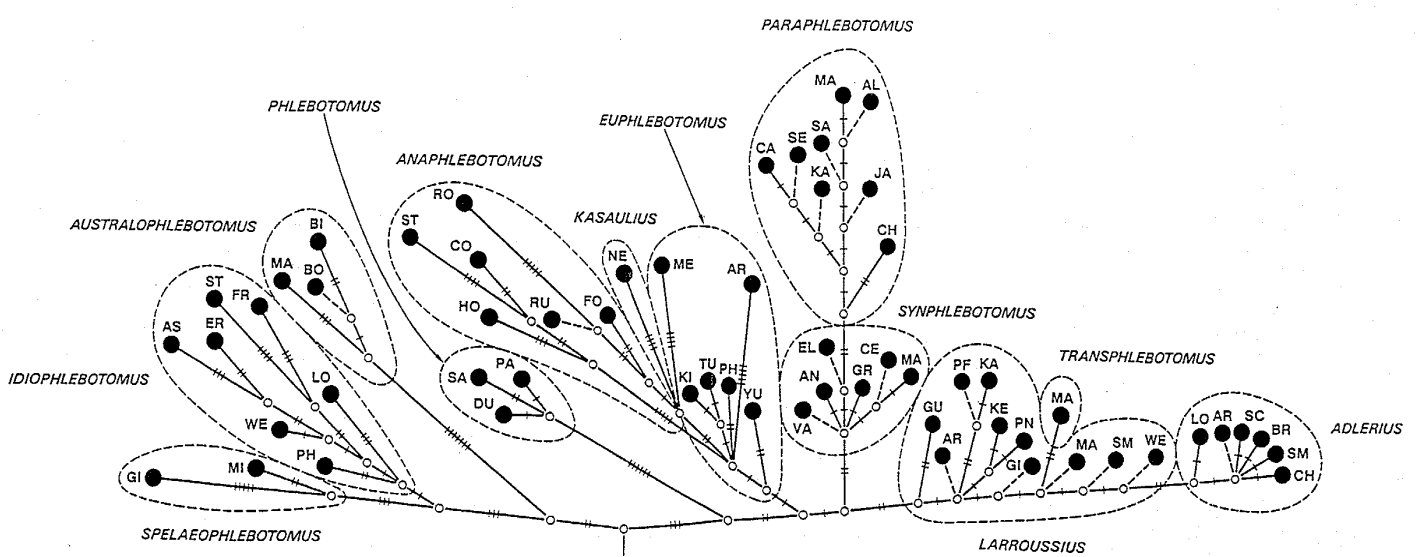

Fig. 3: 59 species of the genus Phlebotomus. Cladistic analysis using 40 characters and 102 states. Most parsimonious cladogram (163 steps). Symbols are the same as in Fig. 1. Bars indicate the number of steps. Elimination of certain characters prevents discrimination between certain taxa: P.(Ph.) bergeroti and P.(Ph.) papatasi; P.(Pa.) andrejevi, P.(Pa.) mongolensis, $P .(P a$.$) nuri,$ P.(Pa.) similis and P.(Pa.) saevus; P.(Sy.) rossi and P.(Sy.) martini; P.(L.) aculeatus, P.(L.) transcaucasicus and P.(L.) perfiliewi; P.(L.) langeroni, P.(L.) longicuspis, P.(L.) longipes, P.(L.) orientalis, P.(L.) pedifer, P.(L.) tobbi and P.(L.) perniciosus; $P .(L$.$) neglectus,$ P.(L.) notus, P.(L.) syriacus, P.(L.) wui and P.(L.) major; P.(Ad.) angustus, P.(Ad.) balcanicus, P.(Ad.) comatus, P.(Ad.) hindustanicus, P.(Ad.) kabulensis, P.(Ad.) salangensis, P.(Ad.) turanicus and P.(Ad.) arabicus; P.(Ad.) halepensis and P.(Ad.) longiductus.

major group within the subgenus Larroussius. It must be noted that the demonstration of this state requires delicate dissection and it is not possible to be sure that this has been carried out with all the species. Moreover, within the genus Phlebotomus, exceptionally long ducts are a synapomorphy of the subgenus Anaphlebotomus. Cylindrical spermathecae, without ornamentation, and with ducts of uniform diameter opening separately are, therefore, very likely to be close to the ancestral spermathecal structure of the group.

Second hypothesis - This analysis of the characters has led to the abolition (or in one case the regrouping) of certain characters. This reduction in the number of 63 characters to 40 , subdivised into 102 states, brings the number of individual species considered to 59. Despite this loss of data at the specific level, the overall structure of the new phylogenetic tree is not seriously altered (Fig. 3). Therefore the abolished characters have little influence on the first phylogenetic hypothesis. Some noticeable differences are, however, to be found. The taxa Spelaeophlebotomus, Idiophlebotomus and Australophlebotomus constitute one of the two initial branches of the tree. All of the known vector species of leishmaniasis are situated on the other branch. The branch Phlebotomus arises very early. Anaphlebotomus emerges from Euphlebotomus in two distinct branches. The subgenera which are probably the most developed, Synphlebotomus and Paraphlebotomus, then Larroussius, Transphlebotomus and Adlerius, divide up in the same way as on the previous tree.

\section{DISCUSSION}

Characters used and methodology - In future cladistic analysis, greater selectivity of characters is required, although the problem of separation of the closely related species will then become acute. Because of the taxonomic and phylogenetic importance of each group of characters, this choice would have to be reasoned and very prudent. Despite the technical balancing carried out, the characters probably do not carry equal weighting in the analysis. Thus, the probably adaptative characters should be accorded lesser evolutionary weighting. On the basis of their supposed development in related groups or at the level of the family, certain character states were considered $a$ priori as ancestral. Some of these "postulats" were clearly invalidated by the cladistic analysis and the study of the characters within the genus. In order to root the phyletic tree, it is therefore necessary to limit the plesiomorphies at the beginning of the cladistic process, to those firmly established at the level of the family. In fact a study limited to the species of the genus Phlebotomus can only give a very partial insight into the development of certain characters in the Phlebotominae or in the Psychodidae as a whole. It is, therefore, essential to extend this study to other genera. Elsewhere, without hypothesizing plesiomorphies, the rooting of the tree by using of an outgroup such as Sergentomyia or another closely related Psychodid should be tried.

Taxonomy and phylogeny - The methods of 
numerical analysis confirm the soundness of classical classifications, while refining the taxonomic levels.

Spelaeophlebotomus and Idiophlebotomus Whilst excluded from the genus Phlebotomus by some authors (Abonnenc 1972, Artemiev \& Neronov 1984), these two taxa are retained as subgenera by others (Lewis et al. 1977, Lewis 1982), in the interests of stability. The phenetic classifications, factorial analysis or ascending hierarchical classifications, show a large distance between these two groups and the other subgenera. Also, the phylogenetic hypotheses show them to emerge precociously on a separate branch. The absence of spatulate sensillae on the palps, a ratio width of wing/gamma greater or equal to 4 , and the long cylindrical styles are ancestral characters common to the species of these two taxa. They all possess three characters for which the developmental direction remains to be determined: long third palpal segments, absence of mesanepisternal setae, and presence of intra-abdominal rods. Moreover Spelaeophlebotomus has an unarmed cibarium and a short gamma, probably plesiomorphic states. Furthermore, is the presence of only four spines on the styles, compensated for by a non deciduous bristle, really a developed state? Most of the species of the group Idiophlebotomus have a cibarial armature. The coxites of some species also have a group of bristles the sub-apical position of which is probably ancestral. Their respective position on the graphs, dendrograms or cladograms, as well as the detailed study of their characters, lead us to believe that Spelaeophlebotomus and Idiophlebotomus merit generic rank.

Australophlebotomus - As with the last two, this subgenus clearly separates from the rest of the genus Phlebotomus. Phenetic analysis always positions it at a distance from the genus cluster, or on a isolated branch of the dendrogram. Initially linked to the subgenus Phlebotomus, it joins Spelaeophlebotomus and Idiophlebotomus on the same branch of the cladogram, after the elimination of certain characters. In the possession of long palpal segments 5 and some mesanepisternal bristles, Australophlebotomus is distinct from Spelaeophlebotomus and Idiophlebotomus. Furthermore, they have a number of characters in a developed state: female antennal formula, spatulate palpal sensillae, short ovoid styles with three spines, etc. Their phenetic distance from the other subgenera and the distinctive development of a number of the characters make Australophlebotomus a clearly separate group which probably merits generic rank.

Phlebotomus - This taxon (only four species) is placed amongst the more primitive subgenera.
It is probably linked to Australophlebotomus. A certain number of characters shared with the known fossil species (morphology of the styles, grouping of the spines, etc.) support this hypothesis. The short spines on the styles and the spines on the lateral lobes are distinctive and probably apomorphic characters. Two other apomorphic characters, the small basal lobes and sub-apical group of bristles on the coxites, have a questionable taxonomic value, as their homology with those of more advanced groups requires further study.

Euphlebotomus and Kasaulius - The interspecific distances within this group are comparatively large. As several distinctive characters of $P$. $(K$.) newsteadi (shape of the halteres, length of the legs, etc.) have not been taken into account, it is impossible totally to isolate this species from Euphlebotomus and, therefore, formally justify the creation of the subgenus Kasaulius Lewis 1982. The position of the two subgenera on the various cladograms suggests they are relatively ancient.

Anaphlebotomus - Specific characters clearly split Anaphlebotomus into two groups, one Asian, the other African. The latter includes $P$. fortunatarum, which is native in the Canary Islands. In fact, the phenetic analysis reveals the probable artificial character of the subgenus. Cladistic analysis indicates that this is relatively ancient subgenus. Despite its lack of unity, it still seems to be the central region of the evolution of the genus. The relatively developed level of the group is, however, emphasised by the morphology of the styles (especially in the Asiatic species), by the presence of four spines (except in P. fortunatarum), and also by the existence of exceptionally long spermathecal ducts which join to form a common duct.

Paraphlebotomus and Synphlebotomus - The phenetic analysis places these closely related subgenera near the subgenus Phlebotomus. On the other hand, the cladistic analysis situates them among the more highly evolved taxa, with recent speciation. The presence of long basal lobes with long bristles and the presence of spatulate tips to the parameres are developed characters common to them both. But the subgenus Paraphlebotomus seems to have evolved separately, developing generally short ovoid styles, with only four spines.

Larroussius, Transphlebotomus and Adlerius The phenetic proximity of these three subgenera is well established and the proliferation of the species within them is probably recent. While it is difficult to confirm the validity of the subgenus Transphlebotomus Artemiev \& Neronov 1984, the taxa P. mascittii and P. canaaniticus, probably subspecies, seem closer to Adlerius than Larroussius. Their separation appears to be later than that of Larroussius, just before the appearance of Adlerius. 
A median group of bristles on the coxites and the long digitiform aedeagus, are developed characters common to all forms in this group. However, the particular case of $P$. (L.) guggisbergi must be noted; it has no group of coxal bristles, but, on each coxite, in the basal position, has a massive structure which is not very prominent, but is covered with bristles. Evolution in Larroussius included the development of a long digitiform extension of the spermathecal "neck". In the major of Larroussius group, as well as in Transphleboto-mus, the spermathecal ducts join to form a common duct. The ratio length of the male genital filaments/length of the genital pump ranges from 3 to 5 in Larroussius and Transphlebotomus, to at least 6.5 in Adlerius. The sub-terminal tubercle on the penis tips appears to be a recent acquisition in Adlerius.

Outlook - Cladistic study should make use of characters not normally used in identification, including internal morphological features as well as larval and pupal characters. After careful selection of the characters, the study should be extended to other genera of the Phlebotominae. A similar process should be applied at the supra-specific level to Old World sand flies, secondarly to Psychodidae as a whole. This would perhaps enable the determination of a universally acceptable taxonomic status of sand flies: Phlebotomidae or Phlebotominae? Comparison of our results with the phenetic and cladistic analysis of biochemical and chromosomal data will make a major contribution to the taxonomy and phylogeny of the Phlebotominae. Eventual synthesis with ecological and behavioural data will allow a biogeographical and bioclimatic study to proceed on a firm basis. New light may be shed on populations and the evolution of characters. A combination of all this information, with parallel studies on Leishmania spp. and their reservoir hosts, will perhaps contribute to a fuller understanding of the structure of leishmaniasis foci.

\section{ACKNOWLEDGMENTS}

To Prof. F Vaillant (Faculté des Sciences, Grenoble), Prof. DM Jarry, Dr DT Jarry, Dr G Lanotte and Mr E Serres (Faculté de Médecine, Montpellier), Prof. RW Ashford (Liverpool School of Tropical Medicine), Dr L
Matile and Dr J-P Hugot (Muséum National d'Histoire Naturelle, Paris) for their valuable advice.

\section{REFERENCES}

Abonnenc E 1972. Les Phlébotomes de la Région Ethiopienne (Diptera, Psychodidae). Mém ORSTOM, Paris 55: 1-289.

Artemiev MM, Neronov VM 1984. Distribution and Ecology of Sandflies of the Old World (genus Phlebotomus), (USRR Com UNESCO Prog Man Biosph), Inst Evol Morphol Anim Ecol, USSR Acad Sci, Moscow: 1-208. [in Russian].

Felsenstein J 1978. The number of evolutionary trees. System Zool 27: 27-33.

Felsenstein J 1985. Confidence limits on phylogenies: an approach using the bootstrap. Evolution 39: 783-791.

Hennig W 1972. Insektenfossilien aus der unteren Kreide. IV. Psychodidae (Phlebotominae), mit einer kritischen Uebersicht über das phylogenetische System der Familie und die bisher beschriebenen Fossilien (Diptera). Stuttg Beitr Naturk 241: 1-69.

Jaccard P 1908. Nouvelle recherche sur la distribution florale. Bull Soc Vaud Sci Nat 44: 223-270.

Killick-Kendrick R, Killick-Kendrick M, Tang Y, Sang DK, Johnson RN, Ngumbi PM 1993. Phlebotomine sandflies of Kenya (Diptera: Psychodidae). I. The validity of Phlebotomus (Larroussius) elgonensis Ngoka, Madel and Mutinga. Ann Trop Med Parasit 87: 207-215.

Lane RP, Alexander B 1988. Sandflies (Diptera: Phlebotominae) of the Canary Islands. $J$ Nat Hist 22: 313-319.

Legendre L, Legendre P 1979. Ecologie Numérique. 2. La Structure des Données Écologiques, Collection d'Ecologie, Masson, Paris, 13, 254 pp.

Lewis DJ 1982. A taxonomic review of the genus Phlebotomus (Diptera: Psychodidae). Bull Br Mus (Nat Hist) (Ent) 45: 121-209.

Lewis DJ, Young DG, Fairchild GB, Minter DM 1977. Proposals for a stable classification of the phlebotomine sandflies (Diptera: Psychodidae). Syst Ent 2: 319-332.

Loew H 1845. Dipterologische Beiträge. 1. Schulprogramm K. Friederich-Wilhems-Gymnasium Posen, Posen, $52 \mathrm{pp}$.

Rondani C 1840. Sopra una Specie di Insetto Dittero. Memoria Prima per Servire alla Ditterologia Italiana $n^{\circ} 1$, Donati, Parma, 16 pp.

Sokal RR, Sneath PHA 1963. Principles of Numerical Taxonomy, Freeman WH and Co, San Francisco, 359 pp .

Wagner WH 1961. Problems in the classification of ferns, p. 841-844. In Recent Advances in Botany, University of Toronto Press, Montreal. 
